\title{
Sustainable Reform of "Introductory Dynamics" Driven by a Community of Practice
}

\section{Prof. Matthew West, University of Illinois at Urbana-Champaign}

Matthew West is an Associate Professor in the Department of Mechanical Science and Engineering at the University of Illinois at Urbana-Champaign. Prior to joining Illinois he was on the faculty of the Department of Aeronautics and Astronautics at Stanford University and the Department of Mathematics at the University of California, Davis. Prof. West holds a Ph.D. in Control and Dynamical Systems from the California Institute of Technology and a B.Sc. in Pure and Applied Mathematics from the University of Western Australia.

\section{Dr. Geoffrey L Herman, University of Illinois, Urbana-Champaign}

Dr. Geoffrey L. Herman is a visiting assistant professor with the Illinois Foundry for Innovation in Engineering Education. He earned his Ph.D. in Electrical and Computer Engineering from the University of Illinois at Urbana-Champaign as a Mavis Future Faculty Fellow and conducted postdoctoral research with Ruth Streveler in the School of Engineering Education at Purdue University. His research interests include creating systems for sustainable improvement in engineering education, promoting intrinsic motivation in the classroom, conceptual change and development in engineering students, and change in faculty beliefs about teaching and learning. He is a recipient of the 2011 American Society for Engineering Education (ASEE) Educational Research and Methods Division Apprentice Faculty Grant. He helps steer the College of Engineering Dean's Strategic Instructional Initiatives Program and consults with the Academy for Excellence in Engineering Education at the University of Illinois. 


\title{
Sustainable Reform of Introductory Dynamics Driven by a Community of Practice
}

\begin{abstract}
The Strategic Instructional Initiatives Program (SIIP) is a college-wide effort to promote the sustainable adoption of evidence-based pedagogies in the College of Engineering. Rather than rely on solitary faculty champions to initiate reforms, SIIP aims to improve the sustainability of reforms by forming Communities of Practice (CoPs) consisting of instructors who are committed to the reform effort. This model of collaborative development provides avenues to secure faculty buy-in, organically spread effective practices, facilitate evaluation, and provide just-in-time training for faculty. This paper describes the rationale for the CoP-based reform model and presents a case study for how this model was used to drive the reform of the course Introductory Dynamics. This course was redesigned to achieve three goals: (1) improve students' low engagement and enthusiasm, (2) improve instructors' experience and reduce their high workload, and (3) maintain and elevate the current standards for content. Introductory Dynamics is one of the core second-year mechanics courses in our engineering curriculum, serving approximately 800 students per year. Course revisions have led to the adoption of a spectrum of evidence-based practices such as context-rich, collaborative problem-solving sessions, and classroom response systems. These efforts have improved student satisfaction with the course and have stimulated additional reform efforts of other high-enrollment mechanics courses.
\end{abstract}

\section{Introduction}

The Strategic Instructional Initiatives Program (SIIP) is a college-wide effort to promote the sustainable adoption of evidence-based pedagogies in the College of Engineering at the University of Illinois at Urbana-Champaign. Rather than rely on solitary faculty champions to initiate reforms, SIIP aims to improve the sustainability of reforms by forming Communities of Practice (CoPs) consisting of instructors who are committed to the reform effort. While these CoPs can be comprised of any number of enthusiastic lecturers and junior faculty, these CoPs are required to also include senior faculty who can broker the departmental buy-in to sustain innovations and reforms. Further, these CoPs are encouraged to meet weekly. During these weekly meetings, the SIIP executive team, comprised of faculty and staff experienced in evidence-based educational methods, facilitate the development and delivery of reforms. This model of collaborative development provides avenues to secure faculty buy-in, organically spread effective practices, facilitate evaluation, and provide just-in-time training for faculty.

As part of the SIIP, one CoP has redesigned the course Introductory Dynamics. This course is one of the core second-year mechanics courses in our engineering curriculum, serving approximately 
800 students per year. Ongoing course reforms aim to achieve three goals: (1) improve students' low engagement and enthusiasm, (2) improve instructors' experience and reduce their high workload, and (3) maintain and elevate the current standards for content.

Prior to reform, the teaching format for Introductory Dynamics involved 3 hours per week of lecture, with one instructor and about 250 students, and 1 hour per week of a discussion section, with 1 teaching assistant and about 30 students. Both lecture and discussion sections were typically taught in a "lecture" style, with the students primarily watching the instructor and taking notes. The content included standard vector dynamics material and focused on derivations and worked problems, with very limited applied examples. The course suffered from low engagement and enthusiasm on the part of both students and instructors. Students routinely reported that Introductory Dynamics was their least-favorite course in the curriculum, and the department faced a constant struggle to persuade faculty to teach the course. Instructors reported that the course was a higher-than-normal teaching workload.

The instructional reforms undertaken in Introductory Dynamics involved the following key changes: (1) an active learning discussion section format, including group work activities and a focus on "real-world" applications of the course material; (2) active learning in lectures using classroom response systems (using the $i>$ clicker system); (3) online content delivery replacing a traditional textbook; (4) online interactive homeworks with immediate feedback (using the SmartPhysics platform); and (5) online help forums to largely substitute for in-person office hours (using the Piazza website).

These evidence-based reforms were supported by a collaborative instructional team and implemented using an innovate-evaluate feedback cycle (see Figure 1). The collaborative instructional team consisted of faculty and lecturers as well as the teaching assistants for the course, and also included faculty who were not teaching the course in the current semester but who were part of the CoP for the course. The teaching assistants received training in group-work facilitation and online student interaction, and collaborated with each other and with instructors in the implementation of reforms.

\section{Communities of Practice for Reform}

The past 15 years have created a surge in research documenting how instructors' implicit epistemologies, beliefs, and commitments drive decision making during instruction and resist productive changes toward evidence-based pedagogies $6 ; 7 ; 11-16$. Unfortunately, the standard methods of dissemination in academia (e.g., articles, workshops, seminars, etc.) are ineffective at changing these belief systems, because these methods are appropriate for technical change efforts rather than adaptive change efforts ${ }^{1 ; 9 ; 10}$. Transformational Learning Theory tells us that technical changes simply require the acquisition of new skills or knowledge to effect change, while adaptive changes inherently challenge core beliefs or epistemologies of the individuals or institutions who are adopting the change ${ }^{10}$. Adaptive changes may be technically simple (e.g., asking more questions in class), but they are difficult to achieve because challenging core beliefs activates a psychological "immune system" that resists change (e.g., asking more questions challenges a primary identity of "instructor as knowledge disseminator") 2;10. Consequently, while "top-down" mandates or isolated development of "best practices" followed by dissemination have proven 


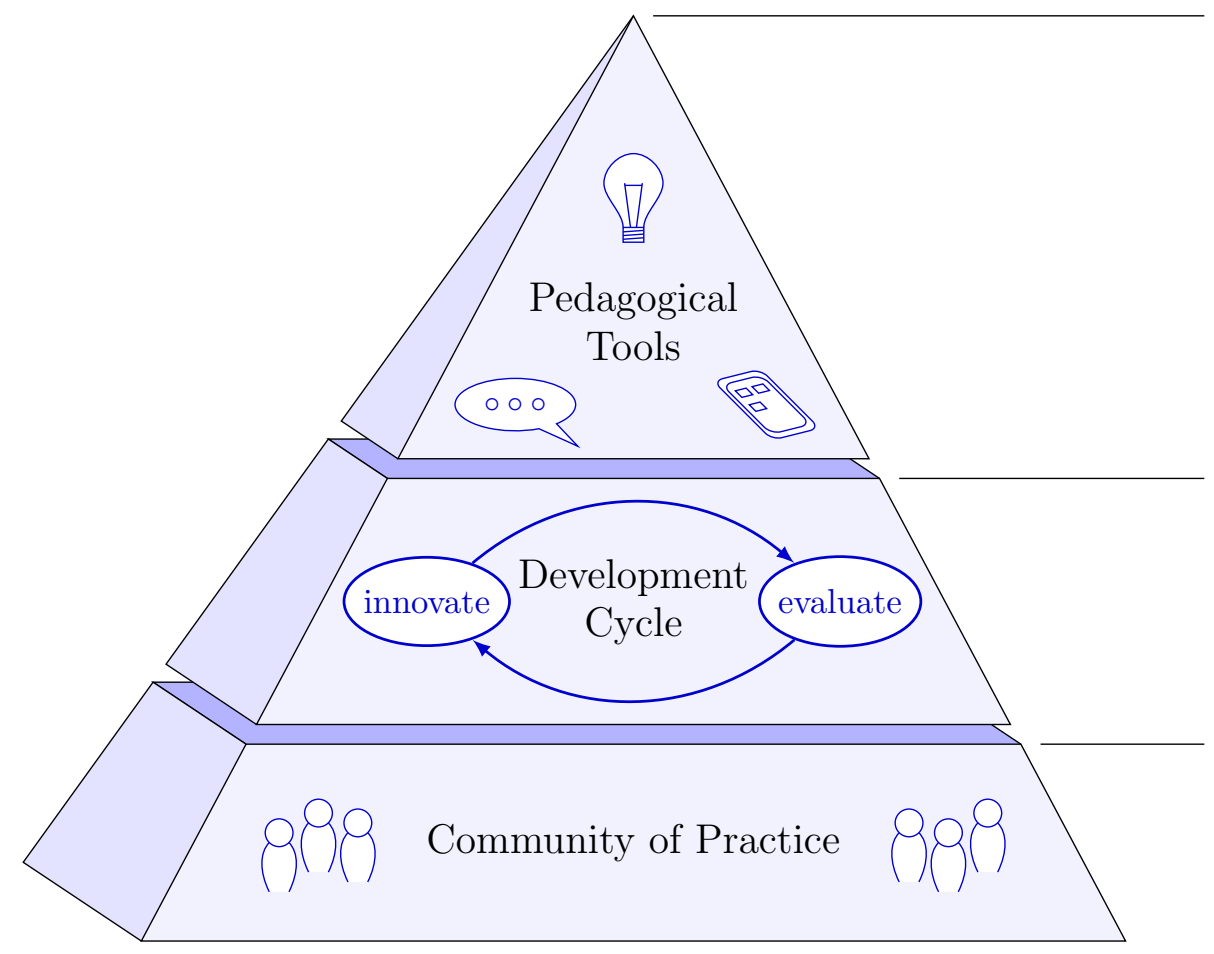

Transformation 3:

Evidence-based tools for state-of-the-art pedagogy in gateway courses.

Transformation 2: Continual improvement via feedback and evaluation.

Transformation 1: Connecting faculty in a community of practice.

Figure 1: The "Pyramid of Change", showing the three levels of transformation that form the objectives of this project; each layer supports the transformations above.

ineffective in promoting the adoption of evidence-based practices ${ }^{1 ; 8}$, long-term strategies that focus on changing faculty beliefs, motivation, and institutional culture have proven effective ${ }^{1 ; 8}$.

Consequently, the SIIP has adopted a long-term strategy focused on creating cultural change. In contrast to large-scale reform efforts that focus on promoting the adoption of specific pedagogies, our model of reform focuses on creating instructional cultures within departments that will foster environments from which adoption of evidence-based pedagogies will emerge. This "culture reform" model relies on a series of three transformations to achieve improvements in instruction. The first transformations support the later transformations (see Figure 1). These transformations are supported by embedding faculty within, or creating new, CoPs focused on creating ongoing, iterative improvements.

Transformation 1: Connecting faculty in Communities of Practice. Teaching traditionally occurs in isolation. Choices of pedagogies and content within a course change as often as the instructors do. This isolationist culture limits the spread of pedagogical advances among faculty and across campus as dissemination relies on secondary efforts divorced from the delivery of the course. In contrast, the goal of SIIP is to create a collaborative teaching culture that will create departmentally or communally owned courses. This collaborative joint ownership of courses provides the cultural basis for creating ongoing, scholarly reform.

Transformation 2: Continual improvement via feedback and evaluation. The isolationist culture also inhibits the ability of faculty to compare and evaluate the advantages of different 


\begin{tabular}{r||l|l|l} 
& Intro. Dynamics & Intro. Solid Mechanics & Intro. Statics \\
\hline \hline Fall 2012 & $\begin{array}{l}\text { Planning and initial } \\
\text { development }\end{array}$ & & \\
\hline Spring 2013 & Initial deployment & $\begin{array}{l}\text { Planning and initial } \\
\text { development }\end{array}$ & \\
\hline \hline Fall 2013 & $\begin{array}{l}\text { Induction of a new } \\
\text { instructor }\end{array}$ & Initial deployment & \\
\hline Fall 2014 & $\begin{array}{l}\text { Induction of another } \\
\text { new instructor }\end{array}$ & $\begin{array}{l}\text { Continuation of new } \\
\text { model }\end{array}$ & $\begin{array}{l}\text { Planning and initial } \\
\text { development }\end{array}$ \\
\hline model & $\begin{array}{l}\text { Induction of a new } \\
\text { instructor }\end{array}$ & Initial deployment
\end{tabular}

Table 1: Timetable of reform development, implementation, and planned future work for the three courses Introductory Dynamics, Introductory Solid Mechanics, and Introductory Statics. Work in 2014 is planned, while work prior to 2014 is completed at the time of writing. See Table 2 for a list of specific reforms.

pedagogies as faculty teach different content, do not collect data, or resist the implications of data that they did not collect. By creating joint course ownership, faculty are able to participate in an implement-evaluate development cycle. This cycle begins by identifying areas for improvement. Then faculty implement targeted reforms and evaluate whether these reforms produce the desired improvements, and then repeatedly iterate the process.

Transformation 3: Organic adoption of evidence-based pedagogies. The collaborative culture and the scholarly implement-evaluate cycle require faculty to engage in ongoing dialogue about how to improve students' learning and experiences. These dialogues allow the CoP to identify areas for improvement and evidence that community members agree are trustworthy, and they allow expertise and beliefs within the group to spread. As faculty agree on areas for improvement and evidence, they can develop consensus on which evidence-based pedagogies to adopt. Further, by creating a CoP of committed faculty, the choice of evidence-based pedagogies will better match the existing departmental culture, promoting acceptance. The CoP rather than an individual then sustains the innovation, distributing the workload and providing mutual support.

\section{A Case Study in Creating Sustainable Reform}

The basis for reform efforts in Introductory Dynamics is a close-knit group of faculty collaborating in a CoP. This group meets weekly both in and out of semester, with meetings being used for reform planning, implementation logistics, evaluation analysis, and general discussions relating to pedagogy and teaching organization. Within the CoP structure, one or two faculty typically take the lead on developing and implementing particular innovations, with the other faculty supporting the effort, providing feedback, and assisting with evaluation. Successful reforms are iterated upon by several faculty, and are codified in written form for the use of new community members, teaching assistants, and faculty in other departments or universities. The implementation timetable is described in Table 1 and a summary of the reforms implemented is shown in Table 2. 


\begin{tabular}{l||l} 
Reform type & List of reforms \\
\hline \hline Online technology & $\begin{array}{l}\text { Online homework system (SmartPhysics), online help/discussion } \\
\text { forum }(\text { Piazza), multimedia online notes, integrated online } \\
\text { gradebooks, online presentation of honors projects. }\end{array}$ \\
\hline In-class technology & $\begin{array}{l}\text { Classroom response system }(\text { i }>\text { clicker }), \text { lectures delivered on tablet } \\
\text { computers, lectures recorded for later online viewing }(\text { Echo360), }\end{array}$ \\
\hline Organization & $\begin{array}{l}\text { Team ownership of courses, TA training and mentoring, per-course } \\
\text { TA weekly meeting, weekly CoP faculty meeting, standardized } \\
\text { office hours location and scheduling, written documentation of } \\
\text { processes and policies, randomized per-student multiple-choice } \\
\text { exams. }\end{array}$ \\
\hline Evaluation & $\begin{array}{l}\text { Standardized mid-semester course surveys, focus group student } \\
\text { feedback, pre- and post-semester concept inventories. }\end{array}$ \\
\hline Other pedagogy & $\begin{array}{l}\text { Active learning in lectures, group work in discussion sections, active } \\
\text { learning in discussions sections, "real-world" applications for } \\
\text { long-form assignments. }\end{array}$
\end{tabular}

Table 2: Summary of reforms implemented in Introductory Dynamics and subsequently extended to other sophomore dynamics courses, following the timetable shown in Figure 1.

A fundamental tenet for the CoP is that the course is "owned" by the team, rather than by whichever faculty happen to be teaching it in a given semester. This represents a dramatic shift from established departmental practice, but is crucial for enabling complex reforms that require multiple semesters to develop, implement, and iterate. Shifting to a team-ownership model required a complex and on-going negotiation between faculty, many of whom have strong opinions on content and pedagogy. The CoP has adopted a flexible model where faculty voluntarily maintain and extend existing course structures, but in which individual faculty still retain freedom regarding implementation in their classes each semester. This "voluntary collaboration" model has proved very successful in maintaining both tight integration between faculty and autonomy of each faculty member.

Over time the CoP has established a high level of trust between faculty, which is key to undertaking risky innovations. This CoP has adopted a highly entrepreneurial methodology, with a preference for fast iteration on new pedagogical styles. For example, the group-work discussion section format was implemented in Spring 2013 with previously-untested worksheets. Initial feedback from students and teaching assistants in the first week indicated that different groups worked at very different speeds, so a system of "optional" questions was implemented for Week 2 that enabled all groups to work for the entire discussion section. Similar iteration on the format details occurred for several weeks until a well-functioning system was established. The CoP was highly supportive during the initial development phase, with all members eager to continue innovation until a good outcome was achieved.

As well as a general agreement on innovation style, the CoP also reached early and sustained agreement on several broad themes. Perhaps most important among these was the role of 
technology, with all CoP members enthusiastically embracing the use of digital technologies both inside and outside the classroom wherever their use facilitated student learning or improved course functioning. Collaboration proved essential in adopting new technology, as there was often a steep initial learning curve and significant iteration required for effective use. For example, the online discussion forum was first piloted using a "best-effort" system for monitoring by teaching assistants. This resulted in irregular monitoring, and students quickly learned that they could not rely on a prompt response, leading to poor overall usage of the forum. The following semester a much more rigorous timetable was enforced for teaching-assistant engagement online, leading to a very positive feedback with student use and resulting in very high usage levels and student satisfaction with the tool. This successful combination of a technology (the online forum) and organization system (teaching-assistant scheduling and training) was then codified in written form and has been successfully implemented in subsequent semesters. The extensive use of online technology, resulting in a blended learning environment ${ }^{3}$, has both directly facilitated student learning as well as reducing instructor workload on menial tasks, allowing for greater emphasis on student-instructor interactions.

An important element for the development of the CoP was support from administration in the college and department at the University of Illinois at Urbana-Champaign. SIIP was instrumental in initiating the reform effort and lead directly to the formation of the CoP, and the formal SIIP process (funding, education, review, etc.) has been extremely helpful in formalizing and rewarding the CoP. The departmental administration has also been highly supportive of the reform efforts, which has been essential for success of the endeavor. For example, the department has ensured that teaching appointments are made consistent with the CoP's plans for course reform, and provided a 50\% increase in the number of teaching assistants to enable the reduction of discussion section sizes from 30 to 20 students as part of the group-work and active-learning discussion format. The support of the departmental and college administrations has required substantial financial investment as well as administrative good-will, but it is hard to imagine how the reform efforts could have succeeded without this support.

\section{Evaluation and Outcomes}

The various reforms were progressively developed and evaluated, with full implementation for Introductory Dynamics in the Spring 2013 semester. Usage and engagement metrics were measured both online and offline, and student opinions and beliefs were measured by a paper survey (89\% response rate) and an in-person focus group conducted by an external evaluator.

The online components of the course had high usage rates, with all students using the online homework system and the other web components having usage rates as shown in Figure 2. Student satisfaction ratings were $95 \%$ for the online homeworks, $87 \%$ for the help web-forum, $85 \%$ for the randomized-exam system, and $96 \%$ for the online course material.

The online-help-forum usage rates varied by student, as shown in Figure 3. While most students used the forum at least once per week on average, a small fraction of students were responsible

for most of the posts, as is common for many online content-creation activities $4: 5$ (sometimes known as the "1\% rule" or "90-10-1" principle). This is captured in Figure 4, which shows the fraction of students responsible for the corresponding fraction of contributions online. Despite the 

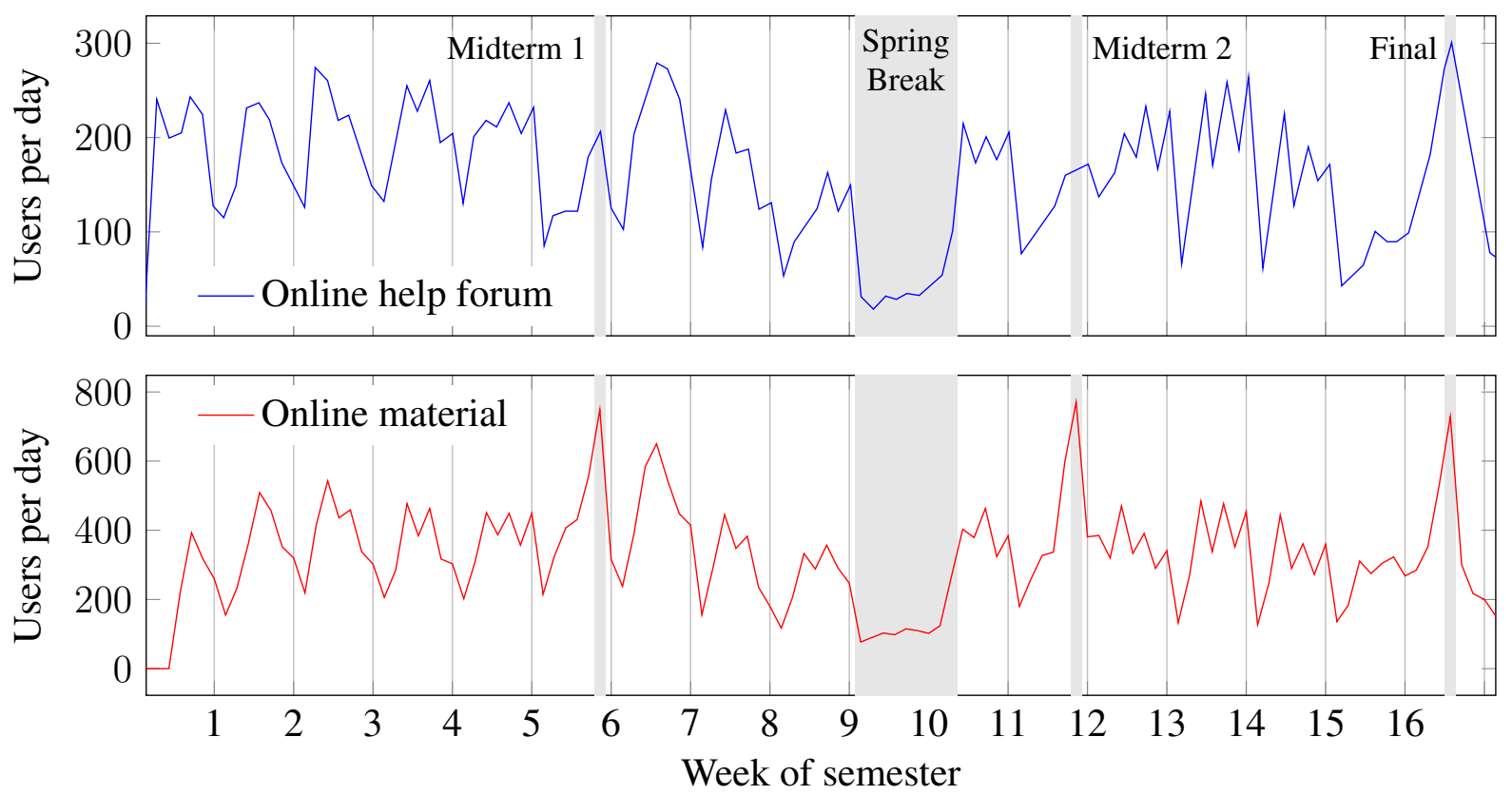

Figure 2: Web site traffic for the online forum (top) and the online course material (bottom) for Introductory Dynamics in the Spring 2013 semester. The vertical grid lines are marked on Fridays, which is when the homework was due each week. Observe that there is not a spike in usage just before homeworks are due. Instead, traffic and activity is evenly spread throughout the week.

fact that $46 \%$ of students never posted to the forum, only $13 \%$ of students were dissatisfied with the forum as a means of interaction and obtaining help, indicating that for a sizable group of students it was sufficient to simply read posting by other students and course instructors.

The use of active learning in both lectures and discussion sections resulted in high attendance rates, as shown in Figure 5. This compares with attendance rates below 50\% in comparable courses. Not only were attendance rates high, but students also found both lectures and discussions to be valuable, with satisfaction ratings of $85 \%$ for lectures and $90 \%$ for discussion sections.

Although the discussion sections were eventually very popular with students, the use of group work in discussions was initially a highly contentious idea, with students organizing complaints and lobbying online and in-person to change back to individual work. By mid-semester, however, student opinion had dramatically shifted, with just $11 \%$ of students expressing a desire to work in an individual setting and an overall $90 \%$ satisfaction rating with discussion sections.

The use of online help forums and group-work discussion sections significantly changed the interaction between the students and teaching assistants (TAs), and was highly successful, with a 96\% student-satisfaction rating for TAs. Additionally, every one of the 11 instructors (9 TAs and 2 faculty) appeared on the student-selected List of Teachers Ranked as Excellent in Spring 2013 (the latest semester with data available at the time of writing), an unprecedented outcome for this course in at least the last 20 years. 

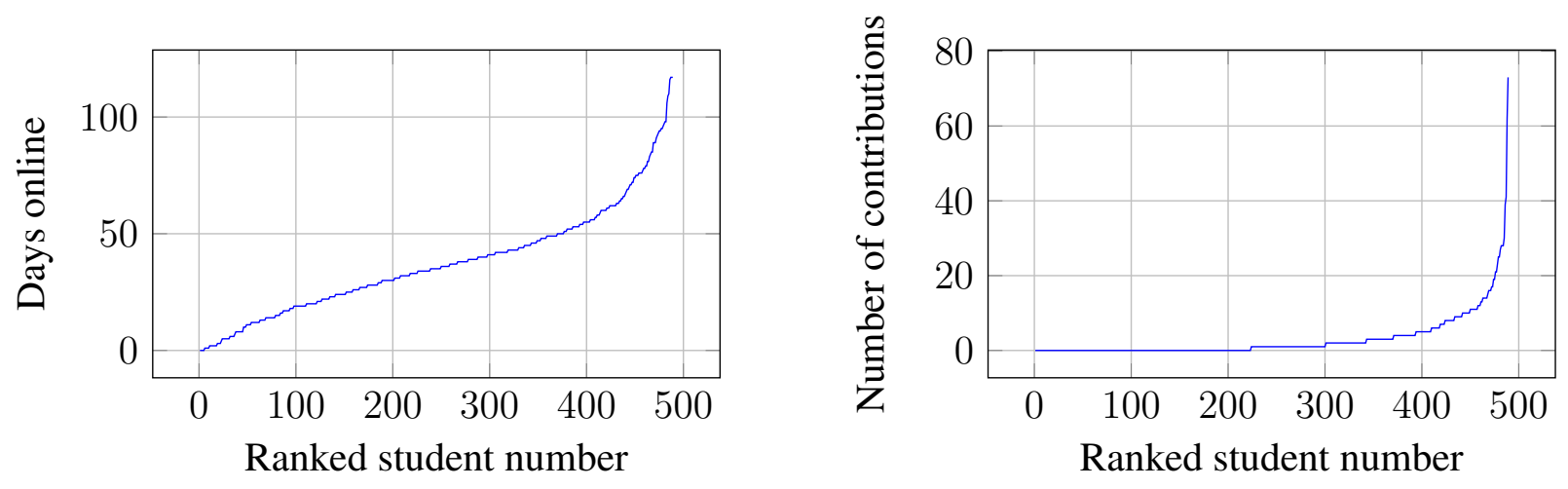

Figure 3: Online-help-forum usage data for Introductory Dynamics in the Spring 2013 semester, showing the number of days online (left) and number of contributions (right) by student number. Contributions include questions, answers, and notes. Observe that $46 \%$ of the students never posted during the semester, but nearly all students logged on repeatedly, with most students logging onto the forum several times per week on average.

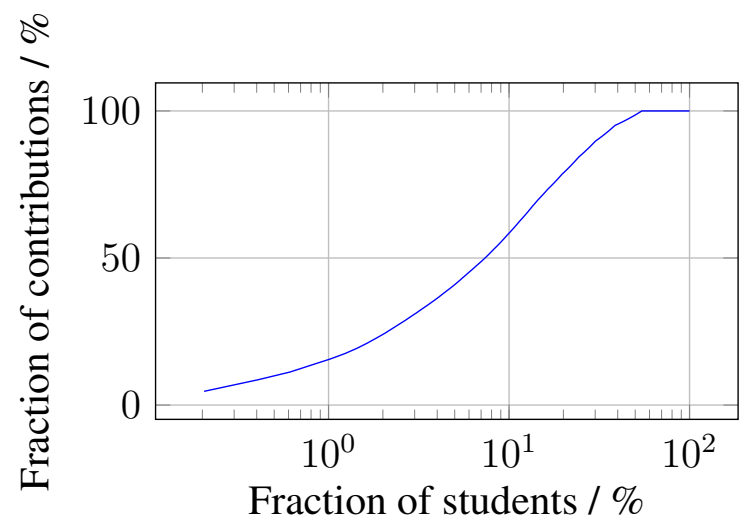

Figure 4: Online-help-forum usage data for Introductory Dynamics in the Spring 2013 semester, showing the fraction of students responsible for each fraction of contributions. Note that the horizontal scale is logarithmic. The individual most-active student made about $5 \%$ of contributions alone; the most active $1 \%$ of students made $16 \%$ of contributions; and the most active $10 \%$ of students made $58 \%$ of contributions. A total of $54 \%$ of students made any contribution at all. 


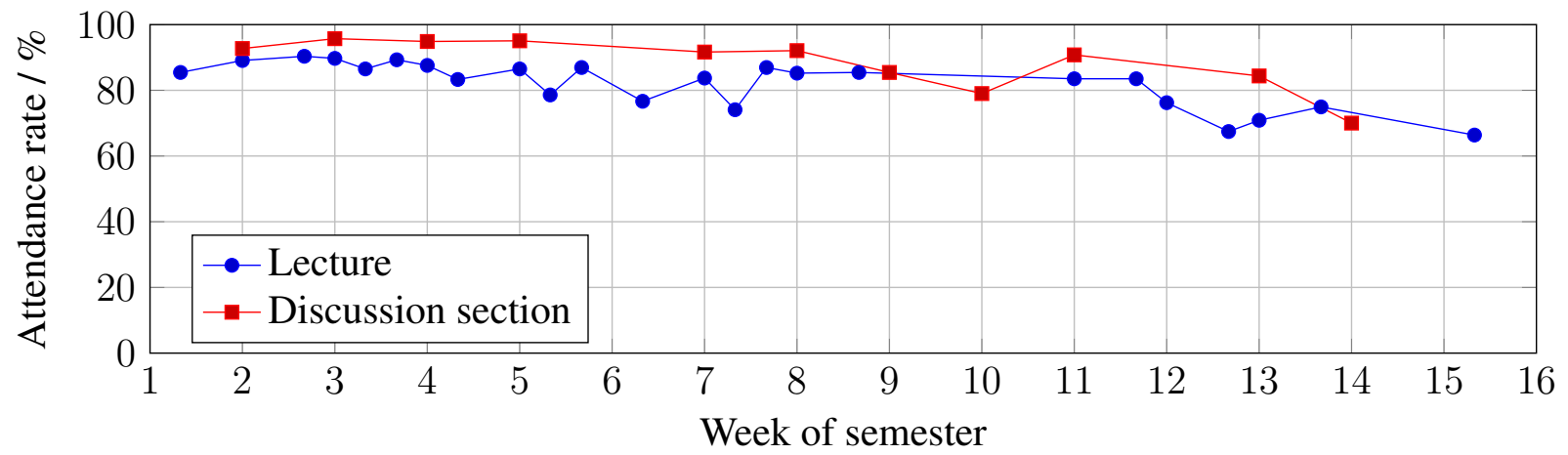

Figure 5: Attendance rates in lectures and discussion sections for Introductory Dynamics in the Spring 2013 semester. The average attendance rate were $82.0 \%$ for lectures and $88.3 \%$ for discussion sections.

\section{Future Work}

At this point, the efforts of the CoP have made significant strides in attaining the first two goals of the reform effort: (1) improve students' low engagement and enthusiasm; and (2) improve instructors' experience and reduce their high workload. The reform efforts in Introductory Dynamics will focus on the third objective of maintaining and elevating the current standards for content. Evaluation of the reform will measure and evaluate students' preparation for future coursework and their learning outcomes.

The superior experience for course instructors produced by these reforms is largely due to the mutual support by faculty within the CoP framework. Consequently, this reform strategy is currently expanding, having been implemented in Introductory Solid Mechanics in Fall 2013, and scheduled for implementation next in Introductory Statics in Fall 2014, as shown in Table 1.

A key challenge for the reform project is the continuation of the CoP. By incorporating all three sophomore mechanics courses into a single CoP, a critical mass of faculty has been established that has sufficient size to be robust against the usual changes in faculty teaching availability. Additionally, the sense of camaraderie engendered by the CoP assists in maintaining engagement. However, it remains to be seen whether recruitment of new faculty into the CoP will continue, and whether the structure is sustainable in the long term.

\section{Reflections and Recommendations}

The formation of a CoP to reform and improve Introductory Dynamics has provided a proof-of-concept for the effectiveness of the CoP model of education reform. Not only has the course improved student and faculty attitudes toward the course, but it has also sparked the reform of additional courses. The reliance on community rather than individuals has sustained the reforms through multiple instructors and multiple semesters.

Although this CoP model has proved effective, it has not necessarily been easy to implement. Of the 13 teams of faculty engaged in SIIP, four have struggled to create anything resembling a CoP. While these faculty clearly desire to improve education, entrenched values of "academic 
freedom" and an individualistic teaching culture impede creation of the CoPs. Similarly, the language of CoPs (even the phrase "community of practice") have served as a barrier to faculty adoption. Careful messaging and careful identification of teachable faculty has been essential.

Despite these new barriers to change, the CoP model has enabled the SIIP to overcome traditional barriers to change. By focusing CoPs around the goal of ongoing improvement of core engineering courses, we were able to engage many faculty who had previously never been involved in faculty development for their teaching. The CoP model provided just-in-time training and apprenticeship for these faculty as they worked alongside more experienced faculty. Similarly, by integrating support personnel within the CoPs, they were able to identify and meet needs as they arose, shortening the feedback cycle and reducing the repetition of mistakes. Similarly, as faculty have engaged in this CoP model, they have become advocates for the model among their peers. This "native" faculty voice, we believe, is ultimately the solution to drawing resistant and hesitant faculty into this new model for education reform.

While garnering this bottom-up support from the faculty CoPs is essential, garnering the top-down support of administration is equally critical. Funding from the Deans has been an essential incentive for inviting faculty into the CoPs and for supplying pedagogical expertise to the CoPs. Departmental administration support through arrangement of teaching schedules, room requests, additional teaching assistant support, and other course logistics is vital to the success of the CoPs. Unless the CoP has input on who will teach a course or where/when the course will be taught, the effectiveness of the CoP can be limited or even negated.

The creation of faculty CoPs offers many avenues for future research on creating sustainable education reform. What are the marks of effective and ineffective faculty CoPs? What type of administrative or cultural support is necessary to create effective CoPs? How do faculty beliefs about teaching and learning change in response to participating in CoPs? Are CoP-driven reforms truly sustainable? How can faculty CoPs be institutionalized to promote their sustainability?

Acknowledgments. This work was supported by the College of Engineering and the Department of Mechanical Science and Engineering at the University of Illinois at Urbana-Champaign as part of the Strategic Instructional Initiatives Program (SIIP).

\section{References}

[1] A. Beach, C. Henderson, and N. Finkelstein. Facilitating change in undergraduate STEM education: Implications from an analytic review of literature. Change: The Magazine of Higher Learning, 44: 52-59, 2012.

[2] S. Brownell and K. Tanner. Barriers to faculty pedagogical change: Lack of training, time, incentives, and tensions with professional identity. CBE-Life Sciences Education, 11:339-346, 2012.

[3] C. R. Graham. Blended learning systems: Definition, current trends, and future directions. In C. J. Bonk and C. R. Graham, editors, The Handbook of Blended Learning, chapter 1, pages 3-21. Pfeiffer, San Franciso, CA, 2006. 
[4] E. Hargittai. Digital na(t)ives? Variation in internet skills and uses among members of the "net generation”. Sociological Inquiry, 80(1):92-113, 2010.

[5] E. Hargittai and G. Walejko. The participation divide: Content creation and sharing in the digital age. Information, Communication \& Society, 11(2):239-256, 2008.

[6] M. Z. Hasweh. Effects of science teachers' epistemological beliefs in teaching. Journal of Research in Science Teaching, 33:47-64, 1996.

[7] C. Heath and D. Heath. Switch: How to Change Things When Change is Hard. Crown Business, 2010.

[8] C. Henderson and M. Dancy. Barriers to the use of research-based instructional strategies: The influence of both individual and situational characteristics. Physical Review Special Topics: Physics Education Research, 3:020102, 2007.

[9] C. Henderson, A. Beach, and M. Famiano. Promoting instructional change via co-teaching. American Journal of Physics (Physics Education Research Section), 77:274-283, 2009.

[10] R. Kegan and L. L. Lahey. Immunity to Change: How to Overcome It and Unlock the Potential in Yourself and Your Organization. Harvard Business Review Press, Cambridge, MA, 2009.

[11] J. A. Luft and G. H. Roehrig. Capturing science teachers' epistemological beliefs: The development of the teacher beliefs interview. Electronic Journal of Science Education, 11:38-63, 2007.

[12] J. Reeve. Why teachers adopt a controlling motivating style toward students and how they can become more autonomy supportive. Educational Psychologist, 44:159-175, 2009.

[13] A. H. Schoenfeld. How We Think: A Theory of Goal-Oriented Decision Making and its Educational Applications. Studies in Mathematical Thinking and Learning Series. Routledge, New York, NY, 2010.

[14] C. Tsai. Nested epistemologies: Science teachers' beliefs of teaching, learning, and science. International Journal of Science Education, 24:771-783, 2002.

[15] C. S. Wallace and N. Kang. An investigation of experienced secondary science teachers' beliefs about inquiry: An examination of competing belief sets. Journal of Research in Science Teaching, 41: 936-960, 2004.

[16] R. Yerrick, H. Parke, and J. Nugent. Struggling to promote deeply rooted change: The "filtering effect" of teachers' beliefs on understanding transformational views of teaching science. Science Education, 81:137-159, 1997. 